\title{
Twisted Intramolecular Charge Transfer (TICT) Controlled by Dimerization: An Overlooked Piece of the TICT Puzzle
}

\author{
Ahmed M. El-Zohry,* Esam A. Orabi, * Martin Karlsson, and Burkhard Zietz \\ Cite This: J. Phys. Chem. A 2021, 125, 2885-2894 \\ Read Online
}

ABSTRACT: Organic dyes have shown high efficiencies in solar cells, which is mainly attributed to the push-pull strategy present in such dyes upon attaching to the semiconductor surfaces. We deeply studied the fundamental photophysical properties of cyanoacrylic dyes, mostly the L1 dye, and found unique emission properties that depend on many factors such as the solvent polarity and the concentration of the dye and could present a complete emission picture about this family of dyes. The L1 dye shows an intramolecular charge transfer (ICT) emission state at low concentrations (approximately nanomolar scale) and shows a twisted intramolecular charge transfer (TICT) emission state in specific solvents upon increasing the concentration to the micromolar scale. Moreover, the associated emission lifetimes of the ICT and TICT states of the L1 dye depend on solvent basicity, highlighting the role of hydrogen bond

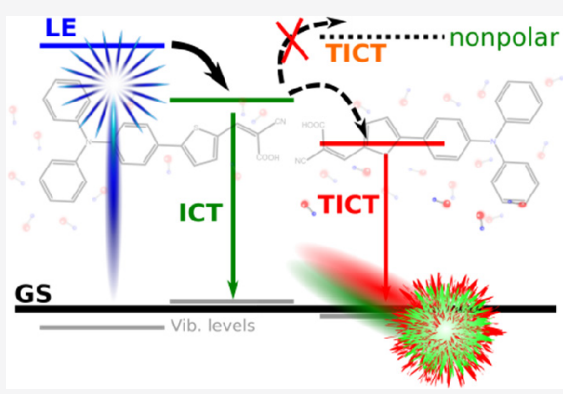
formation on controlling such states. Density functional theory calculations are performed to gain insight into the photophysical properties of the dye and revealed that $\mathrm{H}$-bonding between the carboxylic groups triggers the dimerization at low concentrations. Using femtosecond transient absorption, we assigned the rate of TICT formation to be in the range $(160-650 \mathrm{fs})^{-1}$, depending on the size of the studied cyanoacrylic dye. Therefore, we add herein a new dimension for controlling the formation of the TICT state, in addition to the solvent polarity and acceptor strength parameters. These findings are not limited to the studied dyes, and we expect that numerous organic carboxylic acids dyes show similar properties.

\section{INTRODUCTION}

Numerous donor-acceptor substituted aromatic compounds show dual fluorescence, as first observed by Lippert et al., ${ }^{1}$ and is explained by the twisting of the donor relative to the acceptor group. $^{2-5}$ The twisting process implies a decoupling of the two groups, i.e., donor and acceptor, and leads to considerable charge transfer and a highly polar, chargeseparated state known as the twisted intramolecular charge transfer (TICT) state. ${ }^{3,6}$ This state is formed after populating the Franck-Condon locally excited (LE) state $^{2}$ (see Figure 1a). Twisting around a single bond in the excited state can then lower the molecule's energy, forming the TICT state. From there, the electronic relaxation can occur with emission of fluorescence of a large Stokes shift, leading to a twisted electronic ground state that quickly converts to the relaxed form. Several conditions need to be fulfilled to form the TICT state. The donor and acceptor groups have to be of sufficiently high polarity to allow for an efficient charge separation, and a polar environment is needed to stabilize the formed polar state. Therefore, TICT emission is usually only seen in polar solvents. Twisting involves a decoupling of the $\pi$-systems of the donor and acceptor units, as is typical for a biradicaloid system. The transition dipole strength between the ground and excited state for such a system is expected to be very small. Besides being a fundamental photophysical phenomenon, TICT has found applications that include $\mathrm{pH}$ and ion indicators, $^{7}$ high selectivity for specific ions, ${ }^{5}$ fluorescent probes, ${ }^{8}$ liquid crystals, ${ }^{9,10}$ fluorescent solar collectors, ${ }^{4,11}$ and volume sensing in polymers. ${ }^{12}$ Recently, it was shown that the TICT state stabilizes the charge separation in dye-sensitized solar cells (DSSCs) through small charge recombination rate. ${ }^{4,13-16}$ For solar cell applications, a charge transfer process is designed to move from the donor to the acceptor moiety, forming an intramolecular charge transfer (ICT) state, ${ }^{13}$ which is considered as a primary emissive state. Thus, the ICT state herein corresponds to the LE state in the traditional studied TICT molecules (see Figure 1a). ${ }^{1}$

Herein, we primarily report on a new red-shifted emission from the L1 dye (5-[4-(diphenylamino)phenyl]thiophene-2cyanoacrylic acid) upon concentration variation in various solvents. This dye was designed as a photosensitizer in DSSCs and comprises a triphenylamine as a primary donor and the cyanoacrylic acid (CyA) as a primary acceptor and anchor group (see Figure $1 \mathrm{~b}$ ). Remarkably, the red emission is only observed at higher concentrations, micromolar scale, and in polar solvents and is assigned to the TICT emission from L1

Received: January 23, 2021

Revised: March 17, 2021

Published: April 5, 2021 
(a)

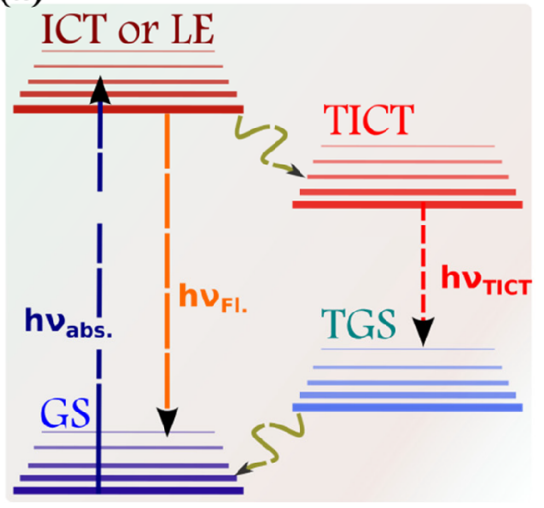

(b)

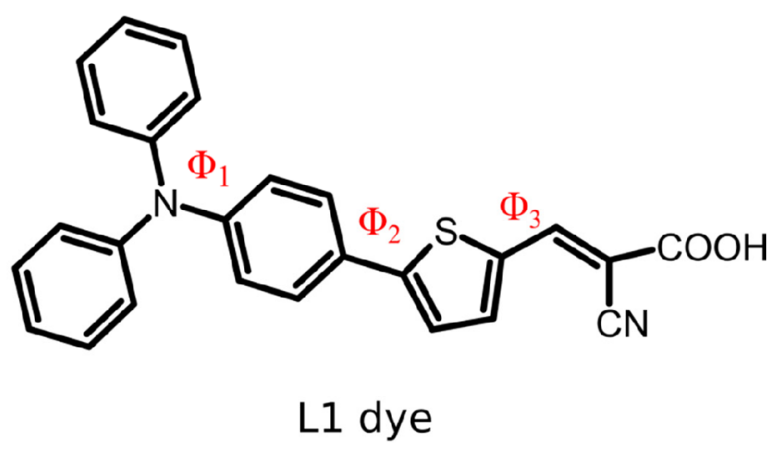

Figure 1. (a) Schematic energy diagram of the TICT state formation populated from LE or ICT state (GS: ground state; TGS: twisted ground state). (b) Chemical structure of the L1 dye, with three main labeled dihedral angles (see the text for more information).
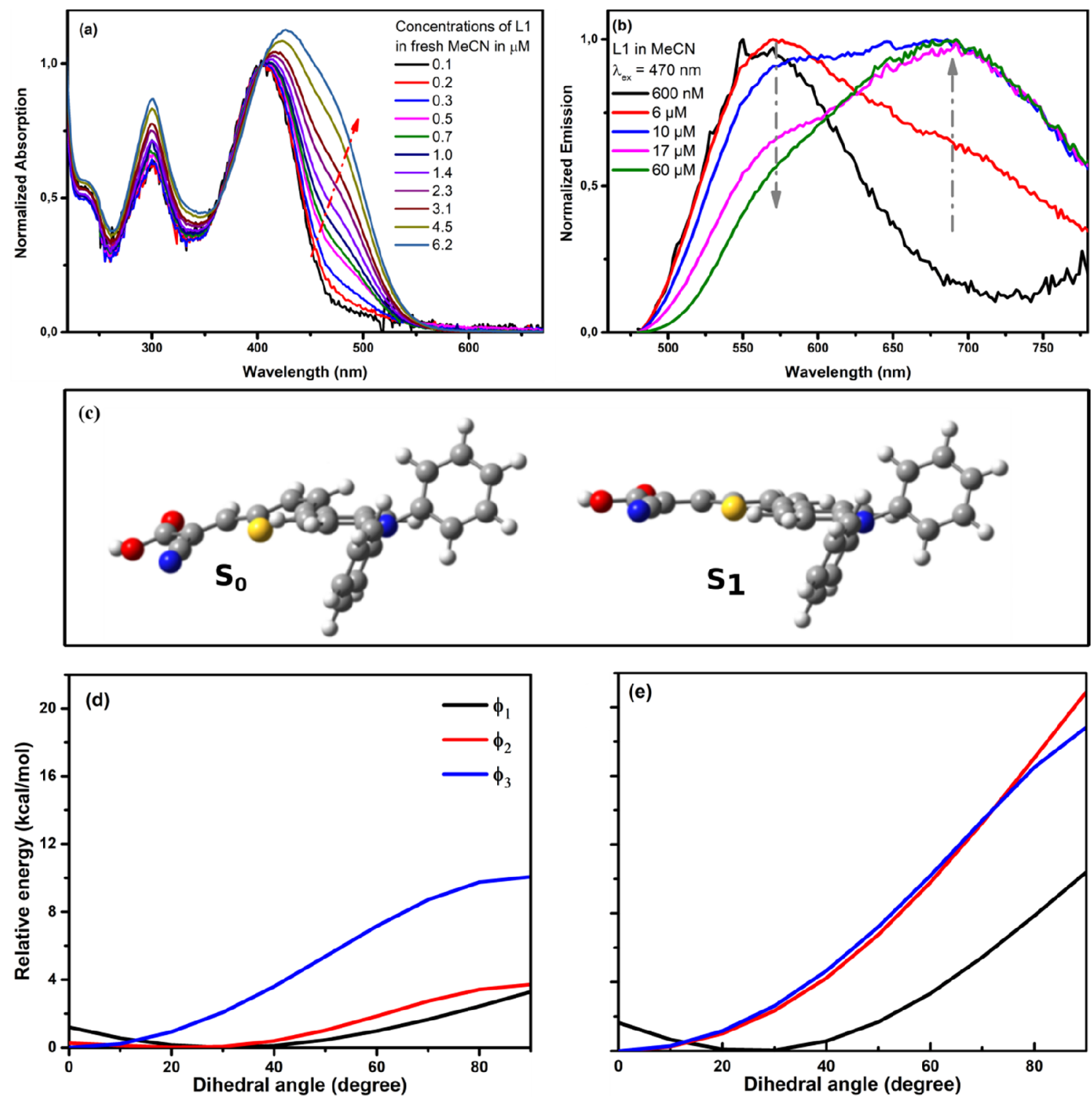

Figure 2. Normalized absorption (a) and emission (b) spectra of the L1 dye in acetonitrile. (c) Global minimum structure of L1 in the $S_{0}$ and $S_{1}$ states calculated with CAM-B3LYP/6-31G(d) in acetonitrile. Atom color code: H (white), C (gray), N (blue), O (red), and S (yellow). Potential energy curves calculated from relaxed scan of $\phi_{1}$ (black), $\phi_{2}$ (red), and $\phi_{3}$ (blue) of L1 in the $S_{0}\left(\mathrm{~d}\right.$ ) and $S_{1}$ (e) states. 
dimers. Noticeably, the emission lifetimes of the TICT state are shorter than the ICT state even if the dimers are present. Density functional theory (DFT) calculations confirm the stability of dimers in solution and the changes in the potential energy surface (PES) at certain dihedral angles of the L1 dye, which support the experimental observations about large-scale motions of the dye in the excited state. Earlier observations of red-shifted emission from dimers of the classical TICT molecule (dimethylamino)benzonitrile (DMABN) and from self-complexes in supersonic jets have been attributed to excimers and not to a TICT state. ${ }^{17}$ The presence of TICT process of cyanoacrylic dyes in solution confirms the previous finding about the role of TICT process to minimize the charge recombination in solar cells. ${ }^{4,16,18,19}$ This is thus the first account of dimer TICT emission for cyanoacrylic dyes in solution, which as well can be extended to other organic dyes with similar acceptor moieties.

\section{METHODS}

Chemicals. The L1 dye (5-[4-(diphenylamino)phenyl]thiophene-2-cyanoacrylic acid (CAS 762269-56-7) was obtained from Dyenamo AB. However, the synthesis of L1 was described earlier. ${ }^{20}$

Steady-State Absorption and Emission. Absorption and emission spectra were performed at a right angle in a $1 \mathrm{~cm}$ cuvette by using a Varian Cary 5000 and an Horiba Jobin Yvon Fluorolog, respectively. Emission measurements were automatically corrected for wavelength-dependent instrument sensitivity.

Time-Correlated Single Photon Counting. The TCSPC setup has been described in detail previously. ${ }^{21}$ Briefly, the sample was excited with a picosecond diode laser (Edinburgh Instruments, EPL405) at $404.6 \mathrm{~nm}$ (77.1 ps pulses), giving an instrument response function of ca. 100 ps.

Streak Camera Measurement. The streak camera setup has been described earlier. ${ }^{21}$ Briefly, excitation of the sample with ultrafast laser pulses was performed by using a frequencydoubled Ti:Sa oscillator (Coherent Mira) output (400 nm). Fluorescence at a right angle to the excitation was passed through a Bruker SPEC 250IS spectrograph and onto the streak camera (Hamamatsu streak camera and blanking unit C5680 in combination with a Synchroscan Unit M5675). The charge-coupled device (CCD) camera (Hamamatsu Orca-ER C4742-95) was used in binning mode $(2 \times 2$ pixels $)$ to give a $512 \times 512$ pixel matrix.

Transient Absorption. A detailed description of the transient absorption setup can be found in a previous publication. $^{22}$ Briefly, the ca. $100 \mathrm{fs}(1 \mathrm{kHz})$ output pulses from a Coherent Legend amplifier were split, and one part was frequency doubled for $400 \mathrm{~nm}$ excitation. Another part of the output was sent into a moving $\mathrm{CaF}_{2}$ plate to produce white light as the probe beam. After passing through the sample and a spectrograph, the light was detected from $\sim 390 \mathrm{~nm}$ to up to $700 \mathrm{~nm}$ by a 512 pixel photodiode array.

Computational Details. Quantum chemical calculations are performed to gain insight into the change of the absorption and emission spectra of L1 in response to its charge (neutral, protonated, and deprotonated) and molecular aggregation (monomer vs dimer). The geometry of $\mathrm{L} 1$ and its deprotonated and various protonated forms was optimized in the ground $\left(S_{0}\right)$ and first excited state $\left(S_{1}\right)$, and the absorption and emission spectra were calculated with the time-dependent DFT method. ${ }^{23}$ These calculations were performed in acetonitrile, described implicitly by using the polarized continuum model (PCM), with the Gaussian 16 program. $^{24}$ Density functional theory (DFT) calculations were performed with the $6-31 \mathrm{G}(\mathrm{d})$ basis set and the CAM-B3LYP functional. ${ }^{25}$ This functional is chosen as it takes long-range interactions into account, which is important to describe ICT states. ${ }^{26}$ Relaxed potential energy scans of the dihedral angles $\phi_{1}, \phi_{2}$, and $\phi_{3}$ of L1 (see Figure 1b) were performed in acetonitrile in the $S_{0}$ and $S_{1}$ states to explore the impact of excitation on molecular twisting and natural bond orbital (NBO) analysis was performed to quantify the degree of intramolecular charge transfer in the two states. Intermolecular interactions and its impact on the absorption spectra of L1 were investigated by using the $(\mathrm{L} 1)_{2}$ dimer as a model. The $\mathrm{S}_{0}$ geometry of the $(\mathrm{L} 1)_{2}$ dimer was optimized in the gas phase starting from various initial binding conformations, and the interaction energies of all stable structures (no imaginary frequencies) are calculated ${ }^{27-29}$ without $(E)$ and with $\left(E^{\mathrm{CP}}\right)$ correction for basis set superposition error (BSSE) by using the counterpoise procedure of Boys and Bernardi. ${ }^{30}$

\section{RESULTS AND DISCUSSION}

The normalized absorption and emission spectra of L1 in acetonitrile are shown in Figure $2 \mathrm{a}, \mathrm{b}$ at various concentrations on the micromolar scale. The maxima of the lowest absorption and emission bands, corresponding to $S_{0}$ and $S_{1}$ at low concentration of L1, are at ca. 405 and $570 \mathrm{~nm}$, respectively. The absorption band is red-shifted, and a new shoulder appears at ca. $470 \mathrm{~nm}$; correspondingly, a new emission band appears at ca. $700 \mathrm{~nm}$ when the concentration of $\mathrm{L} 1$ is increased (Figure 2a,b). The global minimum structures calculated with CAM-B3LYP/6-31G(d) in acetonitrile for L1 in the $S_{0}$ and $S_{1}$ states are reported in Figure $2 c$. In the $S_{0}$ state, $\mathrm{L} 1$ is characterized by a dipole moment $(\mu)$ of $7.3 \mathrm{D}$ and by $30^{\circ}, 26^{\circ}$, and $0^{\circ}$ for $\phi_{1}, \phi_{2}$, and $\phi_{3}$, respectively. In comparison, L1 is more polar $(\mu=12 \mathrm{D})$ and more planar $\left(\phi_{1}=26^{\circ}, \phi_{2}=0^{\circ}\right.$, and $\left.\phi_{3}=0^{\circ}\right)$ in the $S_{1}$ state. NBO analysis reveals charges of 0.17 and 0.28 for the triphenylamine moiety in the $S_{0}$ and $S_{1}$ states, respectively. This indicates that ICT occurs to a greater extent in the excited state, which is consistent with the larger polarity of the $S_{1}$ vs $S_{0}$ state. This ICT is responsible for the observed trigonal planar rather than triagonal-pyramidal geometry of the triphenylamine moiety (Figure 2c). The potential energy curves calculated from relaxed scans of $\phi_{1}, \phi_{2}$, and $\phi_{3}$ of L1 in the $S_{0}$ and $S_{1}$ states are shown in Figure 2 d,e. Besides a switch in $\phi_{2}$ from $26^{\circ}$ to $0^{\circ}$ upon excitation, results show much larger energy barriers for rotations in the excited state. This indicates that structural rearrangements happen before relaxation of the excited L1 molecules.

The new spectral bands that appear upon increasing the L1 concentration are assigned here to the dimers formed between L1 molecules through the CyA groups according to the following simple equation:

$$
\mathrm{L}_{1(\mathrm{M})}+\mathrm{L}_{1(\mathrm{M})} \leftrightharpoons 2 \mathrm{~L}_{1(\mathrm{D})}
$$

in which $\mathrm{M}$ stands for monomer and $\mathrm{D}$ stands for dimer. The dimerization constant based on the absorption changes at 525 $\mathrm{nm}$ by using the modified Benesi-Hildebrand equation ${ }^{31}$ is ca. $9.0 \times 10^{5} \mathrm{M}^{-1}$ (see the fitting in Figure S1). Such a high association constant is matching with the low concentration utilized for such observations. ${ }^{32}$ These spectral changes are not only limited to the L1 dye but are also observed in other 

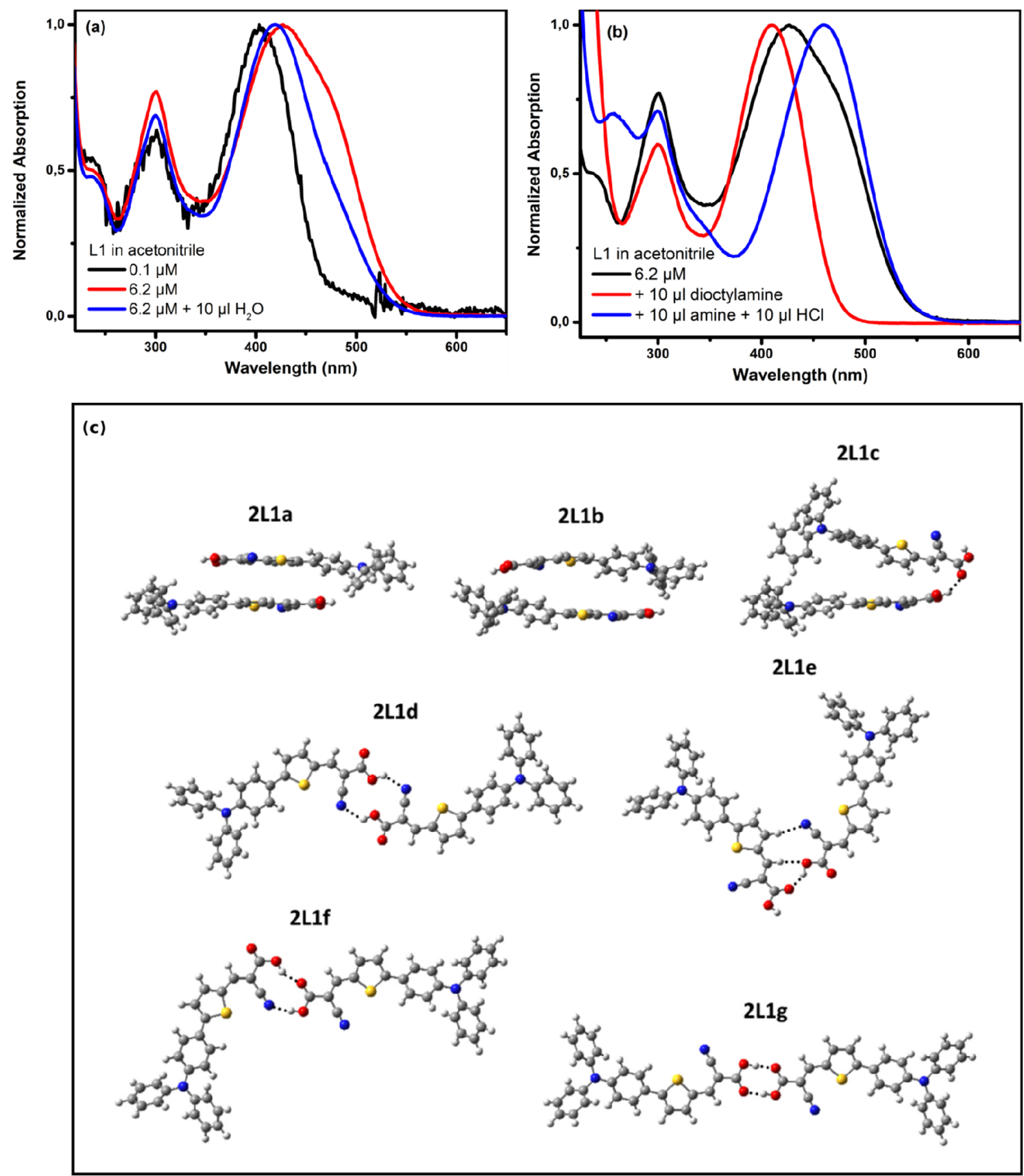

Figure 3. Addition of water (a) and the octylamine base (b) to a solution of $\mathrm{Ll}$ in acetonitrile. Addition of $\mathrm{HCl}$ is also shown after adding the base to the L1 solution. (c) Optimized minimum-energy structures of the $(\mathrm{L} 1)_{2}$ dimer. Structures are arranged from top left to bottom right in order of increasing the interaction energy. Dotted lines connect H-bonded atoms. Atom color code: H (white), C (gray), N (blue), O (red), and S (yellow).

cyanoacrylic dyes with different molecular weights such as the D131 and L0 dyes (see Figures S2 and S3).

We have excluded the possibility of L1 deprotonation at low concentrations by the possible traces of water present in aprotic $\mathrm{MeCN}$ by measuring the absorption spectra of $\mathrm{L} 1$ in the presence of water and in $\mathrm{EtOH}$ (pure protic solvents) (see Figure $3 \mathrm{a}$ and Figure S4). Figure $3 \mathrm{a}$ shows the absorption spectra of $\mathrm{L} 1$ at moderate concentrations of $6 \mu \mathrm{M}$, in which two absorption maxima are detected; the addition of $10 \mu \mathrm{M}$ of water minimizes slightly the band at $470 \mathrm{~nm}$, without complete disappearance. This experiment shows that protic solvents such as water can only disturb the dimer formation.

Interestingly, the equilibrium between monomers and dimers of L1 can be shifted as well by only adding organic bases (Figure $3 \mathrm{~b}$ ) and acids such as $\mathrm{HCl}$ or carboxylic acids (see Figure $1 \mathrm{~b}$ and Figure S5). The addition of the dioctylamine base is expected to deprotonate the $\mathrm{L} 1$, which inhibits the formation of the dimers, giving an absorption spectrum similar to the monomer form of L1 (Figure $3 b$ ). The following addition of excess $\mathrm{HCl}$ to the basic solution of $\mathrm{L} 1$ and the recall of L1's absorption at the dimer position confirms the stability and the reversibility of such an equilibrium dimerization reaction of $\mathrm{L} 1$ (see Figure $1 \mathrm{~b}$ ). The formation of L1 dimers can happen through the carboxylic acid group solely or through the CN group as well. Previously, the crystal structure of 2-CyA showed hydrogen-bonded dimers through the cyano group, ${ }^{33}$ which were also suggested by infrared measurements for a similar CyA-containing dyes. ${ }^{34}$ However, 
addition of carboxylic acids such as acetic acid to L1 results in absorption spectra similar to that of the L1 dimer (Figure S5). In addition, measuring the $\mathrm{LOBr}$ dye (a derivative of $\mathrm{LO}$ with $\mathrm{Br}$ instead of $\mathrm{CN}$ ) yields similar absorption behavior to that of $\mathrm{LO}$ (see Figure S6). These evidences preclude the role of the CN group in the dimerization process itself.

The structures of the various stable conformers of (2L1) calculated with CAM-B3LYP/6-31G(d) in the gas phase are given in Figure 3c, and their interaction energies are reported in Table 1. Structures with stacking arrangements of the

Table 1. Interaction Energy ( $\mathrm{kcal} / \mathrm{mol})$ of the Structures Presented in Figure $3^{a}$

\begin{tabular}{ccc} 
structure & $E$ & $E^{\mathrm{CP}}$ \\
\hline 2L1a & -6.9 & -1.1 \\
2L1b & -11.9 & -4.1 \\
2L1c & -12.7 & -7.2 \\
2L1d & -11.2 & -8.7 \\
2L1e & -13.7 & -11.0 \\
2L1f & -17.6 & -14.4 \\
2L1g & -24.8 & -20.7
\end{tabular}

${ }^{a} E$ and $E^{\mathrm{CP}}$ are interaction energies without and with correction for BSSE.

molecules (2L1a-2L1c) are less stable than those with planar orientation of the interacting pair $(2 \mathrm{~L} \mathbf{1 d}-\mathbf{2 L 1 g})$, indicating that dimerization is attributed to H-bonding rather than hydrophobic association of the aromatic groups. The most stable structure $(\mathbf{2} \mathbf{L} \mathbf{1 g})$ is stabilized by two $(\mathrm{O}-\mathrm{H} \cdots \mathrm{O}=\mathrm{C}) \mathrm{H}$ bonds between the carboxylic groups and display an interaction energy $E^{\mathrm{CP}}=-20.7 \mathrm{kcal} / \mathrm{mol}$. Structure $2 \mathbf{L} \mathbf{1 f}$ is $6.3 \mathrm{kcal} / \mathrm{mol}$ less stable than $2 \mathrm{L1g}$, confirming that dimerization is derived by the carboxylic rather than the cyano group.

The geometry of the most stable conformer $(2 \mathbf{L} 1 \mathrm{~g})$ was also optimized in acetonitrile, and its absorption spectra were calculated (see Figure 4). Results show a slight red-shift in $\lambda_{\max }$ relative to this of the isolated monomer (429 vs $415 \mathrm{~nm}$ ), confirming that the observed increase in $\lambda_{\max }$ with increasing the concentration of L1 (Figure 2a) is a consequence of dimerization.

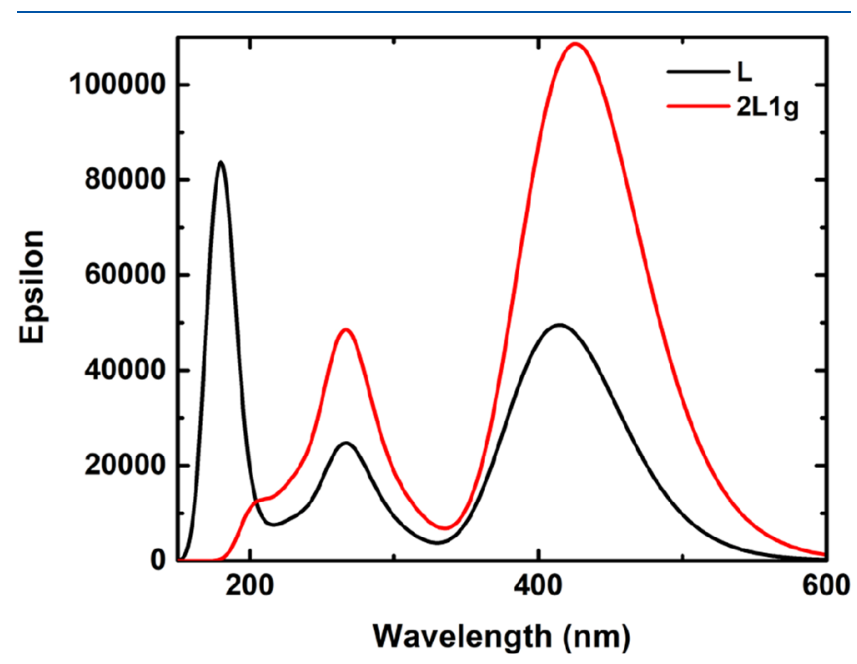

Figure 4. Calculated absorption spectra of L1 and $2 \mathrm{L1g}$ in acetonitrile.
With regard to the emission data of $\mathrm{L1}$, we assign the emission at $570 \mathrm{~nm}$ to the ICT state, in which the charge is transferred from the triphenylamine to the CyA moiety as shown previously by TD-DFT. ${ }^{4,13,35-37}$ However, we attribute the red emission at ca. $700 \mathrm{~nm}$ to a new emission from the TICT state. Because of the low concentration utilized (micromolar) and the polarity of the environment, we excluded the formation of exciplex or excimers.

We have also measured the L1 dye in a wide range of solvents with different properties to correlate between the monomer-dimer equilibrium and the solvent effect on the ICT and TICT states. Figure 5a shows the absorption and emission maxima for $\mathrm{L} 1$ in various solvents of different properties by using dielectric constant herein. As can be seen, in polar solvents, L1 can have different absorption and emission maxima depending on the utilized concentration. At low concentration, in which monomers are expected to be the dominant species, more blue absorption and emission maxima were measured, while at high concentrations of L1 more red spectra were measured representing the dominant dimer state of L1. Simultaneously, the emission lifetime measurements were performed, and different lifetimes associated with different emissive states were found (Figure 5a and Table 3). Interestingly, only the absorption band centered at $675 \mathrm{~nm}$ was seen in formamide $(\varepsilon \approx 115)$, regardless of L1's concentration. This behavior can be explained by complex formation between L1 monomers and the highly polar formamide molecules, which have been confirmed by the titration in $\mathrm{CHCl}_{3}$. Apparently, a highly stable complex species can be formed between formamide and L1 dye (see Figure S7). This reflects the tendency of the cyanoacrylic group to interact with the solvent molecules through hydrogen bonding. ${ }^{32,38}$ Despite, the absence of monomer-dimer equilibria of L1 in formamide, the formamide complex species show as well an emissiontemperature relationship reflecting the presence of an actively excited state process, as shown in Figure $5 \mathrm{~b}$. The emission of L1 in formamide, at low temperature $(77 \mathrm{~K})$, shows an emission increase of 40 times, with a significant blue-shift of the emission band (to $600 \mathrm{~nm}$ ), which has been attributed to the restriction of large-scale motions in the excited state, i.e., the inhibition of the TICT state (Figure 5b).

On the other hand, in nonpolar solvents $\left(\mathrm{CHCl}_{3}\right.$, dioxane, tetrahydrofuran, toluene, and benzene), no changes in absorption and emission bands were observed upon changes in concentration; however, L1 is expected to be in dimeric form (see Figure 5a for absorption and emission maxima in a range of solvents). Thus, $\mathrm{L} 1$ is assumed to exist as dimers in nonpolar solvents, as the positions of the absorption maxima of L1 are close to those of the dimers in polar solvents. Also, nonpolar solvents are expected to interact less with the polar cyanoacrylic groups in $\mathrm{L} 1$ and are thus less likely to break the dimers. The formation of dimers in $\mathrm{CH}_{3} \mathrm{Cl}$ has been confirmed by comparing the absorption spectra after adding a carboxylic acid and an organic base to the solution of $\mathrm{L} 1$ in $\mathrm{CH}_{3} \mathrm{Cl}$ (see Figure S8). The absorption spectrum of $\mathrm{L} 1$ in $\mathrm{CHCl}_{3}$ is slightly changed after adding the carboxylic acid but significantly changed upon adding the organic base, which confirms the presence of the L1 molecules in the dimer form in nonpolar solvents. Thus, Figure 5a shows that $\mathrm{L} 1$ predominantly exists in the dimeric form in solvents with $\varepsilon<20$ but exists in both monomeric and dimeric forms in solvents with $20<\varepsilon<60$. Monomers are, however, the main species in solvents with $\varepsilon>$ 

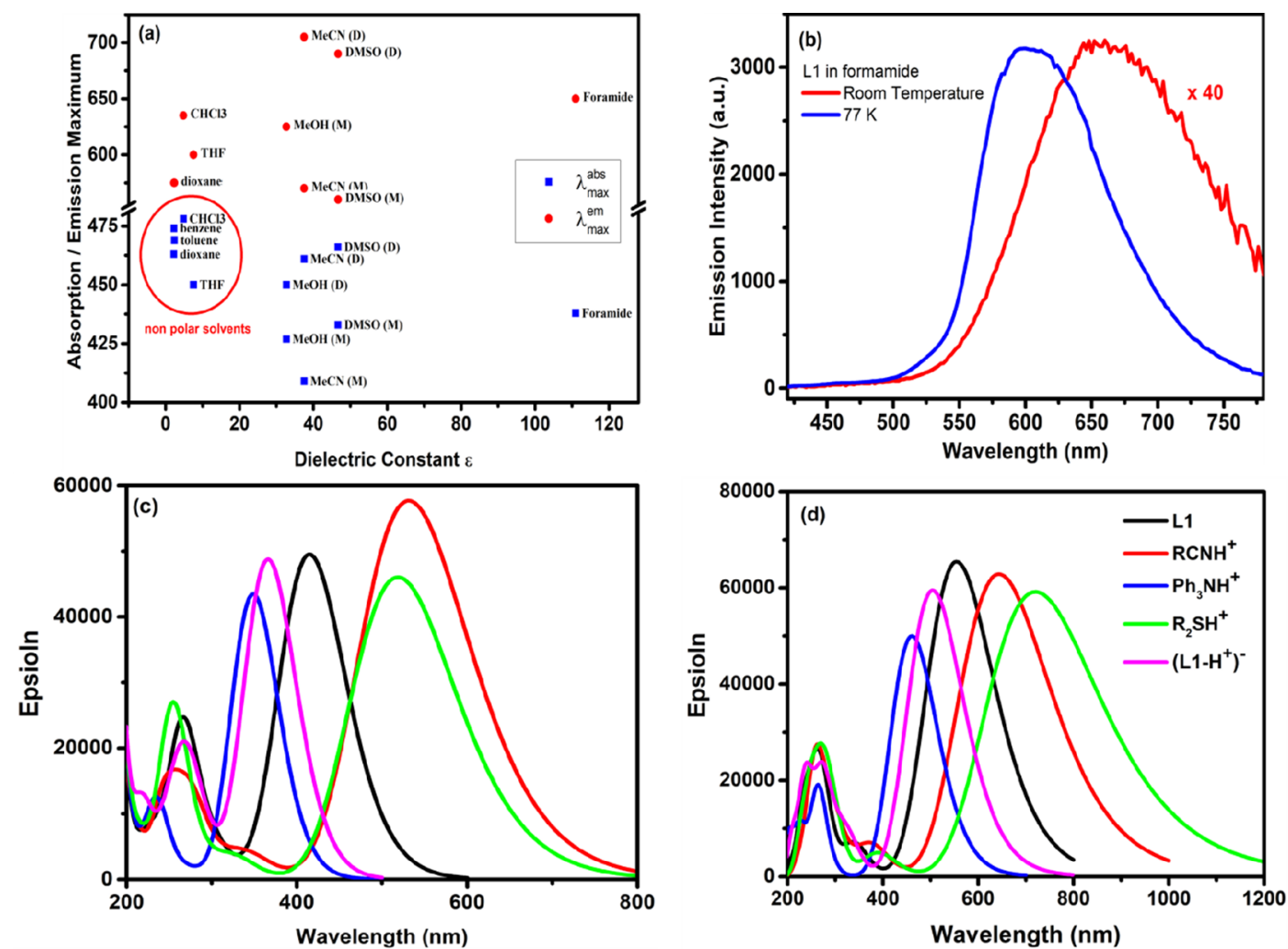

Figure 5. (a) Absorption and emission maxima of L1 in different solvents; M represents monomers, and D represents dimers. (b) L1 emission in formamide at room temperature and at $77 \mathrm{~K}(400 \mathrm{~nm}$ excitation). The emission centered at $675 \mathrm{~nm}$ (TICT) is disabled in the solid state; instead, the normal emission completely dominates (ICT). (c) Calculated absorption and emission (d) spectra of $\mathrm{L}_{1}$ in the neutral (black), protonated $\left(\mathrm{RCNH}^{+}\right.$(red), $\mathrm{Ph}_{3} \mathrm{NH}^{+}$(blue), $\mathrm{R}_{2} \mathrm{SH}^{+}$(green), and deprotonated form $\left(\mathrm{L} 1-\mathrm{H}^{+}\right)^{-}$.
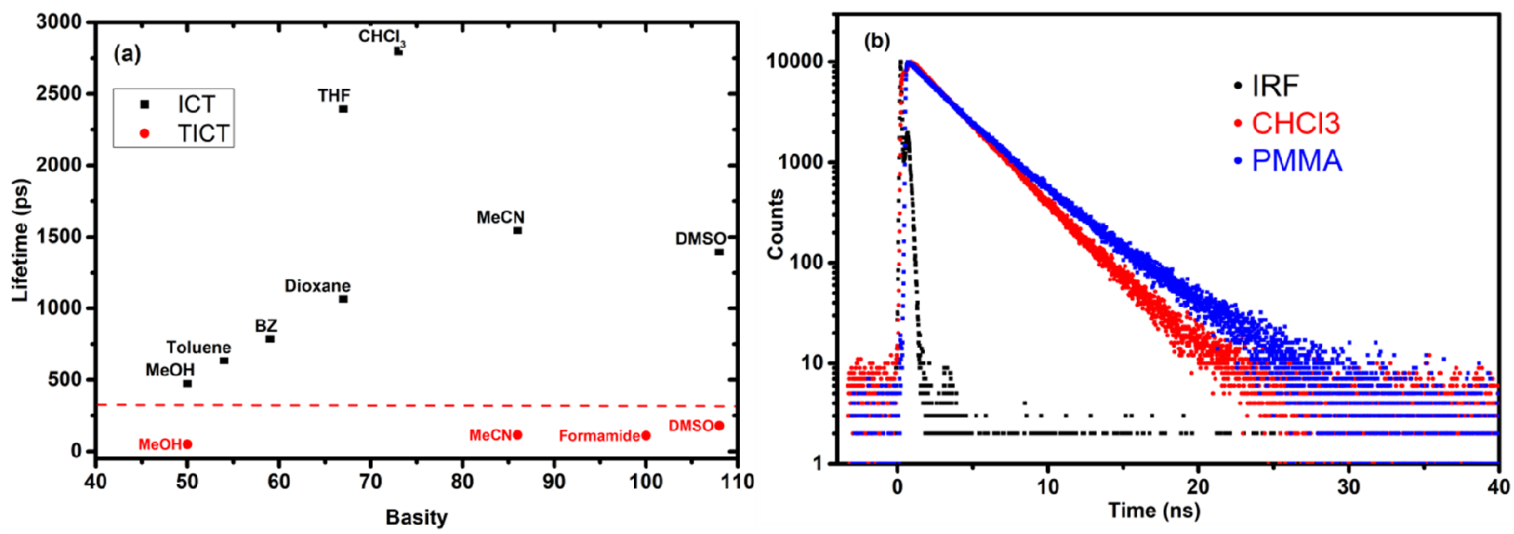

Figure 6. (a) Lifetime of the ICT and TICT states for L1 vs the solvents' basicity. The dotted red line corresponds to the difference between TICT and ICT states. (b) Fluorescence emission intensity of $\mathrm{L} 1$ in $\mathrm{CHCl}_{3}$ and PMMA versus time.

100 due to interaction between L1 monomers and the solvent molecules.

As stated previously, adding organic bases or acids to soluble L1 shows similar effects to the monomer-dimer equilibrium in various solvents. To investigate the impact of strong acids and bases on the absorption and emission spectra of L1, we calculated the absorption and emission spectra of the protonated and deprotonated forms of $\mathrm{L} 1$ in $\mathrm{MeCN}$. We consider the $\mathrm{S}$ and two $\mathrm{N}$ atoms as possible protonation sites in L1. The geometry of these three protonated forms and the geometry of the deprotonated molecule (carboxylate ion) were optimized in the $S_{0}$ and $S_{1}$ states. The calculated absorption and emission spectra of the $\mathrm{L}_{1},\left(\mathrm{~L} 1+\mathrm{H}^{+}\right)^{+}$, and $\left(\mathrm{L} 1-\mathrm{H}^{+}\right)^{-}$ species are reported in Figure 5c,d. Calculations predict a maximum absorption wavelength of the neutral dye (L1) at $415 \mathrm{~nm}$, in very good agreement with the experimental value $(405 \mathrm{~nm})$. The fact that the absorption spectrum of $\mathrm{L} 1$ in the presence of $\mathrm{HCl}$ is red-shifted relative to this of the neutral dye (462 vs $405 \mathrm{~nm}$, Figure 2d) indicates that protonation does not occurs at the triphenylamine $\mathrm{N}$ atom, as this will inhibit the ICT process. In fact, calculations predict that such protonated form has a $\lambda_{\max }=349 \mathrm{~nm}$. In line with experiment, the $\mathrm{RCNH}^{+}$and $\mathrm{R}_{2} \mathrm{SH}^{+}$protonated forms show red-shifted absorption spectra ( $\lambda_{\max }=531$ and $519 \mathrm{~nm}$, respectively). However, the lower basicity of the $\mathrm{R}_{2} \mathrm{~S}$ vs $\mathrm{RCN}$ group and the fact that the $\mathrm{R}_{2} \mathrm{SH}^{+}$structure is $24 \mathrm{kcal} / \mathrm{mol}$ less stable than 
$\mathrm{RCNH}^{+}$indicate that the latter is the protonation form of $\mathrm{L} 1$ in the presence of strong acids such as $\mathrm{HCl}$ and TFA. Similar to experiment, calculations predicts a blue-shifted absorption of the deprotonated species $\left(\lambda_{\max }=366 \mathrm{~nm}\right)$ relative to the neutral molecule. The fact that calculations overestimate and underestimate $\lambda_{\max }$ of the protonated and deprotonated species, respectively, is likely due to the implicit description of the solvent. Charge transfer from and toward the solvent molecules surrounding the protonated and deprotonated species would improve the agreement with the experiment. Calculations predict an emission spectrum of L1 with $\lambda_{\max }=$ $555 \mathrm{~nm}$, in a very good agreement with the experimental value $(570 \mathrm{~nm})$. In comparison, the $\mathrm{RCNH}^{+}$protonated form displays emission spectra with $\lambda_{\max }=645 \mathrm{~nm}$, while the acetate anion possesses $\lambda_{\max }=505 \mathrm{~nm}$. The $\phi_{1}, \phi_{2}$, and $\phi_{3}$ dihedral angles for the $\mathrm{RCNH}^{+}$protonated form in the $\mathrm{S}_{0}$ state are $\left(18^{\circ}\right.$, $\left.5^{\circ}, 0^{\circ}\right)$ and are $\left(29^{\circ}, 5^{\circ}, 0^{\circ}\right)$ in the $S_{1}$ state. As can be seen, the calculations provide more accurate results for the protonated form than the pure L1 dimers (see Figure 4); thus, we correlate the behavior of dimers in solution to the $\mathrm{RCNH}^{+}$ protonated form, as the calculated spectra of the latter provide similar results to the experimental ones. The relaxed excited state of the protonated form is $10 \mathrm{kcal} / \mathrm{mol}$ less than the corresponding relaxed state of the neutral form (see Figure S9). This indicates that the TICT state of the L1 dimer will be more stable than the ICT state of the L1 monomer.

The time-resolved emission data were measured with a streak camera and time-correlated single photon counting (TCSPC) to further characterize the two emission bands. The ICT and the TICT bands were found to be solvent-dependent, showing emission lifetimes of $0.5-3.0 \mathrm{~ns}$ for the ICT state and ca. 50-200 ps for the TICT state (Figure 6a and Table 2). The

Table 2. Lifetimes (in picoseconds) for the ICT and TICT States of L1 in Different Solvents

\begin{tabular}{|lrc|}
\hline solvent & ICT & TICT \\
MeCN & 1550 & 100 \\
$\mathrm{MeOH}$ & 450 & $50^{a}$ \\
DMSO & 1400 & 175 \\
formamide & & 110 \\
dioxane & 1100 & \\
benzene & 790 & \\
toluene & 650 & \\
THF & 2400 & \\
CHCl & 2800 & \\
PMMA & 3500 & \\
\hline
\end{tabular}

${ }^{a}$ The TICT lifetime in $\mathrm{MeOH}$ is below the instrument response function of the SPC setup.

emission lifetimes of $\mathrm{L} 1$ were measured in different solvents at low and high concentrations, and only in polar solvents, except formamide, did the emission lifetimes differ between high and low concentrations. The lifetimes in a wide range of solvents are plotted against the basicity ${ }^{39}$ of the solvent in Figure 6a, showing a nice correlation between the emission lifetimes and solvent basicity. The solvent basicity reflects the solvent property to accept hydrogen bonds. ${ }^{40,41}$ Clearly, the large scale motions of L1, in either ICT or TICT, depend on the H-bond interactions in the excited state. Similar solvent effects have been detected previously but a carboxylic acid dye. ${ }^{32}$ As can be seen, only at high concentrations (dimers) and in sufficiently polar solvents, TICT is enabled as a faster deactivation channel. In nonpolar solvents, such as benzene, the L1 is expected to be in the dimeric form; however, the lifetime measurements are independent of concentration, and the dimers in these nonpolar solvents show long-lived ICT emission. To check the suppression of TICT state in L1, we measured the embedded form of L1 in the PMMA matrix (poly(methyl methacrylate)), and as expected long-lived emission species with lifetime of ca. $3.5 \mathrm{~ns}(81 \%)$ and $1.2 \mathrm{~ns}$ (16\%) were measured. The minor $1.2 \mathrm{~ns}$ component is likely to represent $\mathrm{L} 1$ in a slightly different environment, possibly interacting with specific groups of the PMMA. ${ }^{39}$ Comparing the lifetime emission of $\mathrm{L} 1$ embedded in PMMA and in $\mathrm{CH}_{3} \mathrm{Cl}$ confirms the inability of dimers in nonpolar solvents to populate efficiently the TICT state. The $3.5 \mathrm{~ns}$ component is assumed to be close to the radiative lifetime of L1 and a sign of a complete inhibition of the TICT formation. Similarly, the TICT formation could be completely prevented in frozen formamide by lowering the temperature to $77 \mathrm{~K}$ (see Figure $5 b)$. These measurements also highlight the fast conversion between LE and ICT state in L1, as the stiff conditions in PMMA could not prevent the population of ICT state. Thus, our mixing herein between the LE state and the ICT state is plausible.

To monitor the spectral changes of emission for L1, the transient emission of L1 in DMSO is shown herein, as it shows the most striking spectral changes with variation of concentration. At low concentration (monomer case), the main emission centered at $550 \mathrm{~nm}$ is assigned primarily to the ICT state of monomers (Figure 7, top). However, the emission is not homogeneous, and an emission tail is clearly visible between ca. 650 and $720 \mathrm{~nm}$. Kinetic traces were fitted at 700 $\mathrm{nm}$, and a lifetime of ca. 215 ps was obtained. At $550 \mathrm{~nm}$, two

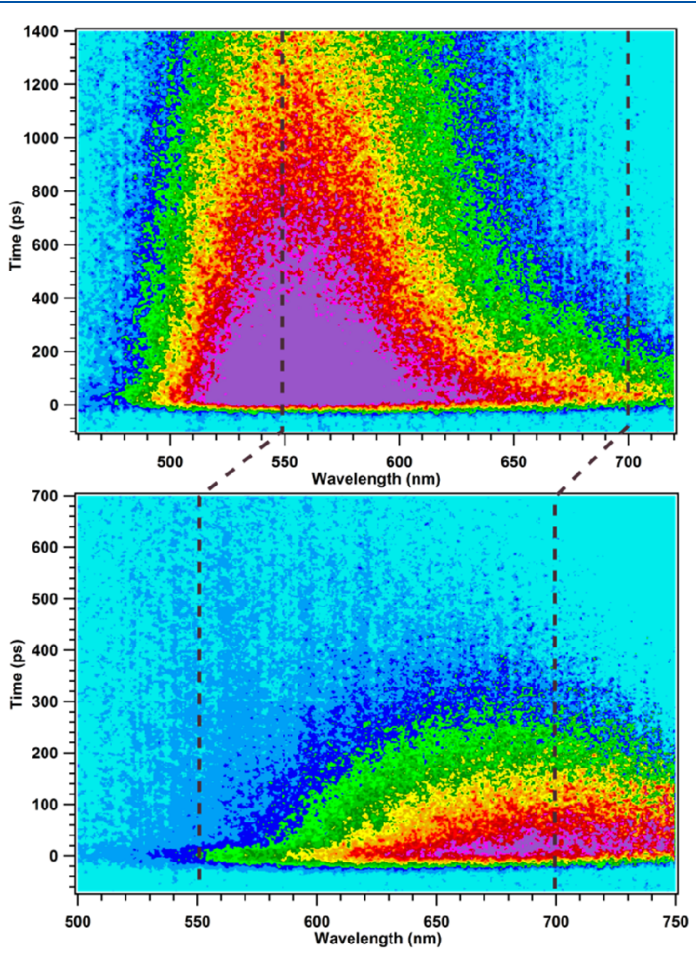

Figure 7. 2D-false emission plot of L1 in DMSO at $200 \mathrm{nM}$ (top) and $20 \mu \mathrm{M}$ (bottom). Mind the different time scales in ps. The dotted black lines indicate to the emission spectral shifts due to the emission states; check the text for more information. 
a.

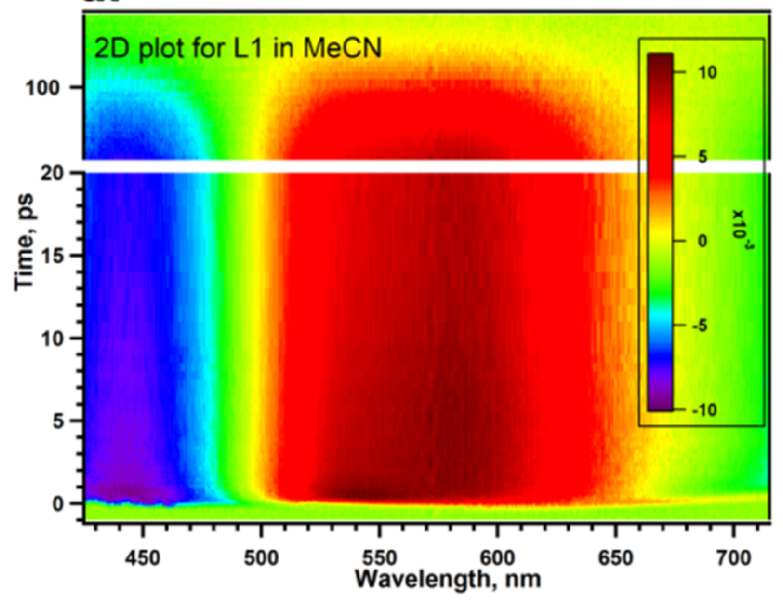

b.

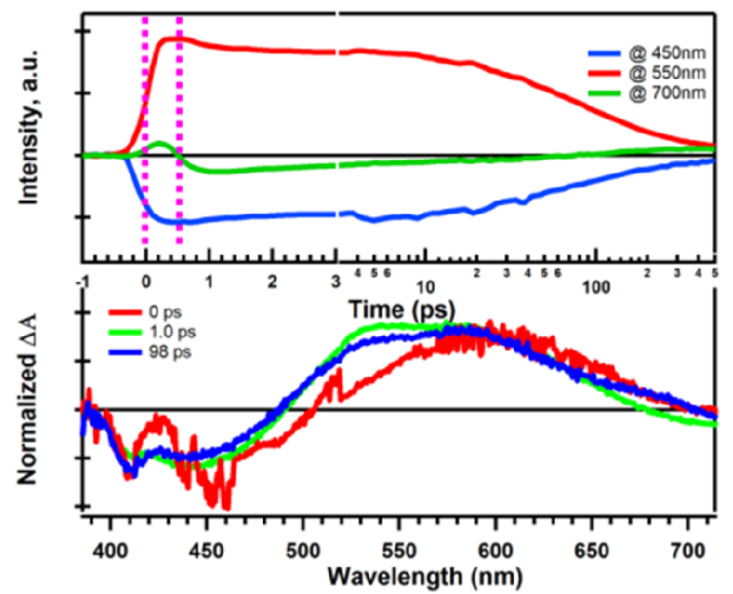

Figure 8. (a) Difference absorption of L1 in acetonitrile after excitation at $400 \mathrm{~nm}, 2 \mathrm{D}$-color plot. (b) Extracted kinetics and spectra. The two dotted pink colors show the delay of the emission related to the ESA due to the TICT state formation. See text for more information.

lifetimes of ca. 300 ps and 1.4 ns were obtained (the latter from TCSPC). We assign the 200-300 ps component to red-shifted emission from the TICT state. At high concentration, the ICT emission band has nearly completely vanished, and the TICT emission band is centered on $650 \mathrm{~nm}$ (see Figure 7, bottom). Fitting by a global procedure gave two lifetimes, ca. 3 ps for the solvent dynamics of $\mathrm{DMSO}^{42}$ and 175 ps for the decay of the TICT state.

Femtosecond transient absorption spectroscopy (TA) was used to characterize the transition between the initially excited ICT state and the TICT state according to the scheme shown in Figure 1. The results for L1 in acetonitrile at high concentration (dimers) after excitation at $400 \mathrm{~nm}$ are seen in Figure 8a,b. The ground state bleach (GSB) in the region around $450 \mathrm{~nm}$ could be fitted with two lifetimes of 1.6 and 97 ps, while for the regions dominated by excited-state absorption (ESA, $550 \mathrm{~nm}$ ) and $700 \mathrm{~nm}$ (ESA + stimulated emission), three lifetimes are needed in the fits, including an additional fast component of 160-350 fs. This fast lifetime component, which is only visible in the excited state and not the GSB, is attributed to the transformation of the ICT state to the TICT state, which then shows stimulated emission at long wavelengths (ca. $700 \mathrm{~nm}$ ). This fast lifetime is also associated with spectral blue shifts in the ESA region from $600 \mathrm{~nm}$ to ca. 525 $\mathrm{nm}$. The lifetimes at different wavelengths are summarized in Table 3. Similar observations were found for the TA of D131

Table 3. Lifetimes for $\mathrm{L} 1$ in Methanol from Fits of Kinetic Curves at Given Wavelengths

\begin{tabular}{clll} 
& \multicolumn{3}{c}{ lifetimes (amplitudes) } \\
\cline { 2 - 4 } wavelength $(\mathrm{nm})$ & \multicolumn{1}{c}{$\tau_{1}(\mathrm{ps})$} & \multicolumn{1}{c}{$\tau_{2}(\mathrm{ps})$} & $\tau_{3}(\mathrm{ps})$ \\
450 & & $1.6(-10 \%)$ & $97(-90 \%)$ \\
550 & $0.16(-58 \%)$ & $0.68(22 \%)$ & $98(30 \%)$ \\
700 & $0.35(64 \%)$ & $1(-25 \%)$ & $92(-11 \%)$ \\
\hline
\end{tabular}

dye (another cyanoacrylic dye) in $\mathrm{MeCN}$ at high concentrations. After the population of the ICT state of D131 dye, a blue-shift in the ESA was observed due to the formation of the TICT state with a formation lifetime of ca. 650 fs (Figure S10). The larger formation lifetime in D131 than in L1 can be assigned to the larger molecular size of D131. Similar correlations between the dye molecule size and such largescale motions have been discussed previously. ${ }^{15}$

The observations from absorption and fluorescence spectroscopy as well as from DFT calculations are summarized in Scheme 1. Upon excitation of the cyanoacrylic dye (L1), the

Scheme 1. Schematic Representation for the Reaction Pathways in the Excited State of L1 upon Changing Solvent Polarity along with the Presence of Monomer Dimer Species in the Solution

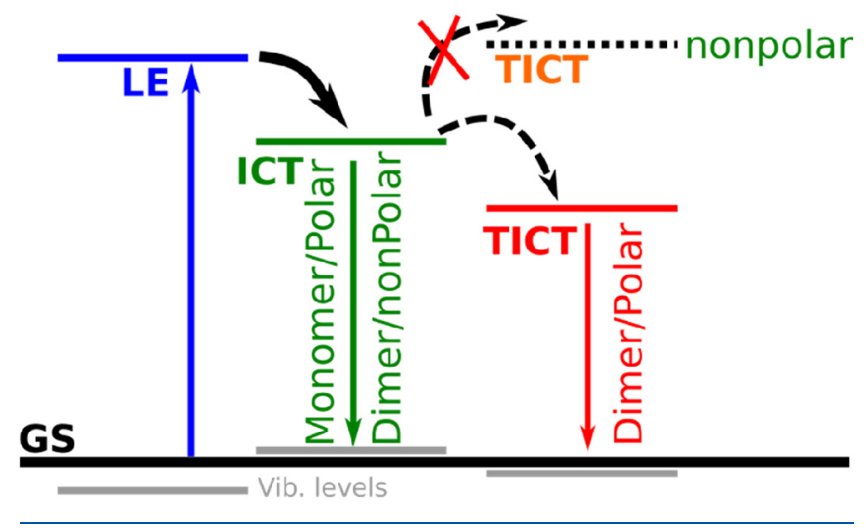

LE state directly populates the ICT that is normally the emissive state; the TICT state is not accessible for the monomer of L1 and for dimers in nonpolar solvents due to an energy barrier present. However, the dimer formation in polar solvents as well as protonation increases the acceptor strength and leads to a lowering of the energy of the TICT state relative to the ICT state, which becomes the lowest emission state, which is characterized by spectral and kinetic changes in the emission properties. Thus, through fine-tuning of the L1 concentration, both emissive states can be detected.

It is worth mentioning to compare between the nature of TICT for L1 in solution and on mesoporous surfaces. We previously interpreted that presence of the TICT state in the L1 on semiconductor surfaces hinders the charge recombination process, ${ }^{4,14}$ indicating that the TICT state should have long-lived emission that the ICT state. This apparent contradiction can be understood via the degree of freedom that the molecules have in solution and not on semiconductor 
surfaces or in the single crystals. In either way, it is clear now that further increase of the acceptor strength of the cyanoacrylic group by dimerization in polar solvents or by attaching it to a semiconductor surface will facilitate the population of the TICT state, leading to red emission in polar solvents and minimize the charge recombination in solar cells.

\section{CONCLUSION}

We have studied the photophysical properties of cyanoacrylic dyes in solution taking the L1 dye as model for this class of organic dyes utilized primarily in solar cells as photosensitizers. Our study revealed the presence of monomer/dimer equilibrium with high association constant. The dimerization has been confirmed experimentally and theoretically to be through the carboxylic acid group. The formation of the L1 dimer modifies the strength of the acceptor group and thereby shifts the energy level of the two lowest excited states (ICT and TICT) in polar solvents such that TICT is stabilized sufficiently to become the lowest and fluorescent state. This opens possibilities for tuning the emission across the visible spectrum solely by changes in dye's concentration. Also, we could correlate the high performance of cyanoacrylic dyes in solar cells due to the population of the TICT state through increasing the acceptor strength of the cyanoacrylic dye., ${ }^{414}$ Thus, our study contributes to both fundamental and applicable understandings for such organic dyes.

\section{ASSOCIATED CONTENT}

\section{SI Supporting Information}

The Supporting Information is available free of charge at https://pubs.acs.org/doi/10.1021/acs.jpca.1c00629.

Figures S1-S10: the presence of monomer-dimer equilibrium for other cyanoacrylic dyes, the effect of adding acids or bases on the absorption and emission data, as well as the fs-TA for the D131 dye in MeCN showing the conversion form the ICT to the TICT state (PDF)

\section{AUTHOR INFORMATION}

\section{Corresponding Authors}

Ahmed M. El-Zohry - Department of Chemistry - Angström Laboratory, Uppsala University, SE-75120 Uppsala, Sweden; Department of Physics - AlbaNova Universitetscentrum, Stockholm University, SE-10691 Stockholm, Sweden; • orcid.org/0000-0003-2901-5815; Email: amfzohry@ yahoo.com, ahmed.elzohry@fysik.su.se

Esam A. Orabi - Department of Chemistry, University of Manitoba, Winnipeg, Manitoba R3T 2N2, Canada; (1) orcid.org/0000-0002-5338-9203; Email: orabiesam@ gmail.com

\section{Authors}

Martin Karlsson - Applied Physical Chemistry, KTH Royal Institute of Technology, SE-10044 Stockholm, Sweden

Burkhard Zietz - Department of Chemistry - Ångström Laboratory, Uppsala University, SE-75120 Uppsala, Sweden

Complete contact information is available at:

https://pubs.acs.org/10.1021/acs.jpca.1c00629

\section{Notes}

The authors declare no competing financial interest.

\section{ACKNOWLEDGMENTS}

Prof. L. Kloo (KTH), Prof. Ivo Leito (University of Tartu), and Prof. R. Lomoth (Uppsala University) are acknowledged for insightful discussions and critical comments.

\section{REFERENCES}

(1) Lippert, E.; Lüder, W.; Moll, F.; Nägele, W.; Boos, H.; Prigge, H.; Seibold-Blankenstein, I. Umwandlung Von Elektronenanregungsenergie. Angew. Chem. 1961, 73, 695-706.

(2) Grabowski, Z. R.; Rotkiewicz, K.; Rettig, W. Structural Changes Accompanying Intramolecular Electron Transfer: Focus on Twisted Intramolecular Charge-Transfer States and Structures. Chem. Rev. 2003, 103, 3899-4032.

(3) Rettig, W. Photoinduced Charge Separation Via Twisted Intramolecular Charge Transfer States. Top. Curr. Chem. 1994, 169, 253-299.

(4) El-Zohry, A. M.; Karlsson, M. Gigantic Relevance of Twisted Intramolecular Charge Transfer for Organic Dyes Used in Solar Cells. J. Phys. Chem. C 2018, 122, 23998-24003.

(5) Lin, Q.; Liu, X.; Wei, T.-B.; Zhang, Y.-M. TICT-ICT State Change Mechanism Based Acetate Fluorescent Sensor Act as an "Off-on-Off" Switch and Logic Gate. Sens. Actuators, B 2014, 190, $459-463$.

(6) Rettig, W. Charge Separation in Excited States of Decoupled Systems-Tict Compounds and Implications Regarding the Development of New Laser Dyes and the Primary Process of Vision and Photosynthesis. Angew. Chem., Int. Ed. Engl. 1986, 25, 971-988.

(7) Rettig, W.; Lapouyade, R. Fluorescence Probes Based on Twisted Intramolecular Charge Transfer (TICT) States and Other Adiabatic Photoreactions. In Topics in Fluorescence Spectroscopy; Lakowicz, J. R., Ed.; Kluwer: New York, 1994; Vol. 4, pp 109-150.

(8) Rettig, W. Photoinduced Charge Separation Via Twisted Intramolecular Charge-Transfer States. In Topics in Current Chemistry; Springer: 1994; pp 253-299.

(9) Friscic, T.; MacGillivray, L. R. Single-Crystal-to-Single-Crystal [2 + 2] Photodimerizations: From Discovery to Design. Z. Kristallogr. Cryst. Mater. 2005, 220, 351-363.

(10) Hoyer, T.; Tuszynski, W.; Lienau, C. Ultrafast Photodimerization Dynamics in Alpha-Cyano-4-Hydroxycinnamic and Sinapinic Acid Crystals. Chem. Phys. Lett. 2007, 443, 107-112.

(11) Rettig, W. Nachr. Chem. Technol. Lab. 1991, 39, 298.

(12) Al-Hassan, K. A.; Rettig, W. Free Volume Sensing Fluorescent Probes. Chem. Phys. Lett. 1986, 126, 273-279.

(13) Debnath, T.; Maity, P.; Lobo, H.; Singh, B.; Shankarling, G. S.; Ghosh, H. N. Extensive Reduction in Back Electron Transfer in Twisted Intramolecular Charge-Transfer (TICT) Coumarin-DyeSensitized $\mathrm{TiO}_{2}$ Nanoparticles/Film: A Femtosecond Transient Absorption Study. Chem. - Eur. J. 2014, 20, 3510-3519.

(14) El-Zohry, A. M.; Cong, J.; Karlsson, M.; Kloo, L.; Zietz, B. Ferrocene as a Rapid Charge Regenerator in Dye-Sensitized Solar Cells. Dyes Pigm. 2016, 132, 360-368.

(15) El-Zohry, A. M. The Origin of Slow Electron Injection Rates for Indoline Dyes Used in Dye-Sensitized Solar Cells. Dyes Pigm. 2019, 160, 671-674.

(16) El-Zohry, A. M.; Alturki, A.; Yin, J.; Mallick, A.; Shekhah, O.; Eddaoudi, M.; Ooi, B. S.; Mohammed, O. F. Tunable Twisting Motion of Organic Linkers Via Concentration and Hydrogen-Bond Formation. J. Phys. Chem. C 2019, 123, 5900-5906.

(17) Lommatzsch, U.; Gerlach, A.; Lahmann, C.; Brutschy, B. Supersonic Jet Studies on the Photophysics of 4-Dimethylaminobenzonitrile (DMABN) and Related Compounds: On the Origin of the Anomalous Fluorescence of Dmabn Clusters in a Supersonic Jet. J. Phys. Chem. A 1998, 102, 6421-6435.

(18) Debnath, T.; Maity, P.; Lobo, H.; Singh, B.; Shankarling, G. S.; Ghosh, H. N. Extensive Reduction in Back Electron Transfer in Twisted Intramolecular Charge-Transfer (TICT) Coumarin-DyeSensitized $\mathrm{TiO}_{2}$ Nanoparticles/Film: A Femtosecond Transient Absorption Study. Chem. - Eur. J. 2014, 20, 3510-3519. 
(19) Verma, S.; Ghosh, H. N. Tuning Interfacial Charge Separation by Molecular Twist: A New Insight into Coumarin-Sensitized $\mathrm{TiO}_{2}$ Films. J. Phys. Chem. C 2014, 118, 10661-10669.

(20) Hagberg, D. P.; Marinado, T.; Karlsson, K. M.; Nonomura, K.; Qin, P.; Boschloo, G.; Brinck, T.; Hagfeldt, A.; Sun, L. Tuning the Homo and Lumo Energy Levels of Organic Chromophores for Dye Sensitized Solar Cells. J. Org. Chem. 2007, 72, 9550-9556.

(21) El-Zohry, A.; Orthaber, A.; Zietz, B. Isomerization and Aggregation of the Solar Cell Dye D149. J. Phys. Chem. C 2012, $116,26144-26153$.

(22) Petersson, J.; Eklund, M.; Davidsson, J.; Hammarström, L. Ultrafast Electron Transfer Dynamics of a $\mathrm{Zn}$ (II)Porphyrin-Viologen Complex Revisited: S2 Vs S1 Reactions and Survival of Excess Excitation Energy. J. Phys. Chem. B 2010, 114, 14329-14338.

(23) Runge, E.; Gross, E. K. U. Phys. Rev. Lett. 1984, 52, 997.

(24) Frisch, M. J.; Trucks, G. W.; Schlegl, H. B.; Scuseria, G. E.; Robb, M. A.; Cheeseman, J. R.; Scalmani, G.; Barone, V.; Mennucci, B.; Petersson, G. A.; et al. Gaussian 16, Rev. A03; Gaussian Inc.: 2016.

(25) Yanai, T.; Tew, D. P.; Handy, N. C. A New Hybrid ExchangeCorrelation Functional Using the Coulomb-Attenuating Method (Cam-B3lyp). Chem. Phys. Lett. 2004, 393, 51-57.

(26) Pastore, M.; Mosconi, E.; De Angelis, F.; Grätzel, M. A Computational Investigation of Organic Dyes for Dye-Sensitized Solar Cells: Benchmark, Strategies, and Open Issues. J. Phys. Chem. C 2010, 114, 7205-7212.

(27) Orabi, E. A. Molecular Dynamics Investigation of the Structural Flexibility of $\mathrm{H}_{2} \mathrm{O}_{2}$ and $\mathrm{H}_{2} \mathrm{~S}_{2}$ in Response to Medium Polarity. J. Mol. Liq. 2021, 329, 115469.

(28) Orabi, E. A.; English, A. M. Modeling Shows that Rotation about the Peroxide $\mathrm{O}-\mathrm{O}$ Bond Assists Protein and Lipid Functional Groups in Discriminating between $\mathrm{H}_{2} \mathrm{O}_{2}$ and $\mathrm{H}_{2} \mathrm{O}$. J. Phys. Chem. $B$ 2021, 125, 137-147.

(29) Orabi, E. A.; Faraldo-Gómez, J. D. New Molecular-Mechanics Model for Simulations of Hydrogen Fluoride in Chemistry and Biology. J. Chem. Theory Comput. 2020, 16, 5105-5126.

(30) Boys, S. F.; Bernardi, F. J. M. P. The calculation of small molecular interactions by the differences of separate total energies. Some procedures with reduced errors. Mol. Phys. 1970, 19, 553-566.

(31) Benesi, H. A.; Hildebrand, J. H. A Spectrophotometric Investigation of the Interaction of Iodine with Aromatic Hydrocarbons. J. Am. Chem. Soc. 1949, 71, 2703-2707.

(32) El-Zohry, A. M.; Zietz, B. Concentration and Solvent Effects on the Excited State Dynamics of the Solar Cell Dye D149: The Special Role of Protons. J. Phys. Chem. C 2013, 117, 6544-6553.

(33) Shklover, V. E.; Struchkov, Y. T.; Kandror, I. I.; Gol'ding, I. R.; Gololobov, Y. G. Crystal Structure of A-Cyanoacrylic Acid. J. Struct. Chem. 1991, 32, 269-272.

(34) Kitamura, T.; Ikeda, M.; Shigaki, K.; Inoue, T.; Anderson, N. A.; Ai, X.; Lian, T.; Yanagida, S. Phenyl-Conjugated Oligoene Sensitizers for $\mathrm{TiO}_{2}$ Solar Cells. Chem. Mater. 2004, 16, 1806-1812.

(35) Hagberg, D. P.; Marinado, T.; Karlsson, K. M.; Nonomura, K.; Qin, P.; Boschloo, G.; Brinck, T.; Hagfeldt, A.; Sun, L. Tuning the Homo and Lumo Energy Levels of Organic Chromophores for Dye Sensitized Solar Cells. J. Org. Chem. 2007, 72, 9550-9556.

(36) El-Zohry, A. M.; Roca-Sanjuan, D.; Zietz, B. Ultrafast Twisting of the Indoline Donor Unit Utilized in Solar Cell Dyes: Experimental and Theoretical Studies. J. Phys. Chem. C 2015, 119, 2249-2259.

(37) Narashima, N.; Mataga, N. Molecular Interactions of P-(N,NDimethylamino)-BenzonitrileElectrostatic Self-Complex and HeteroComplex Formation in the Ground and Excited States. Bull. Chem. Soc. Jpn. 1973, 46, 3016-3020.

(38) El-Zohry, A. M.; Agrawal, S.; De Angelis, F.; Pastore, M.; Zietz, B. Critical Role of Protons for Emission Quenching of Indoline Dyes in Solution and on Semiconductor Surfaces. J. Phys. Chem. C 2020, 124, 21346-21356.

(39) El-Zohry, A. M.; Orthaber, A.; Zietz, B. Isomerization and Aggregation of the Solar Cell Dye D149. J. Phys. Chem. C 2012, 116, 26144-26153.
(40) Waghorne, W. E.; O'Farrell, C. Solvent Basicity, a Study of Kamlet-Taft and Gutmann Dn Values Using Computationally Derived Molecular Properties. J. Solution Chem. 2018, 47, 16091625.

(41) Catalan, J.; Palomar, J.; Diaz, C.; dePaz, J. L. G. On Solvent Basicity: Analysis of the Sb Scale. J. Phys. Chem. A 1997, 101, 51835189.

(42) Horng, M. L.; Gardecki, J. A.; Papazyan, A.; Maroncelli, M. Subpicosecond Measurements of Polar Solvation Dynamics Coumarin-153 Revisited. J. Phys. Chem. 1995, 99, 17311-17337. 